This manuscript is a preprint that is currently undergoing peer-review in the Journal of Natural Gas Science and Engineering. Subsequent versions of this manuscript may have different content. If accepted, the final version of this manuscript will be available via the Peer-reviewed Publication DOI link on the right-hand side of this webpage. We welcome feedback addressed to frmale@utexas.edu 


\section{Comparison of permeability predictions on cemented \\ 2 sandstones with physics-based and machine learning \\ 3 approaches}

$4 \quad$ Frank Male*1, Jerry L. Jensen ${ }^{2}$, Larry W. Lake ${ }^{1}$

5 1. Hildebrand Department of Petroleum and Geosystems Engineering, University of Texas

6 at Austin 2. Bureau of Economic Geology, University of Texas at Austin.

7 * frmale@utexas.edu

\section{Abstract}

9 Permeability prediction has been an important problem since the time of Darcy. Most

10 approaches to solve this problem have used either idealized physical models or empirical

11 relations. In recent years, machine learning (ML) has led to more accurate and robust, but

12 less interpretable empirical models. Using 211 core samples collected from 12 wells in the

13 Garn Sandstone from the North Sea, this study compared idealized physical models based

14 on the Carman-Kozeny equation to interpretable ML models. We found that ML models

15 trained on estimates of physical properties are more accurate than physical models. Also,

16 the results show evidence of a threshold of about $10 \%$ volume fraction, above which pore-

17 filling cement strongly affects permeability. 


\section{Introduction}

19 Sandstone is one of the most common types of reservoir rocks, contributing approximately

$2030 \%$ to the stratigraphic total of sedimentary rocks (Pettijohn, 1975). It is the lithology for

21 eight of the ten largest gas fields in the world (Walsh and Lake, 2003; Sandrea, 2005).

22 Therefore, it is of interest to predict the reservoir properties of sandstones. This paper will

23 focus on analyzing the factors that influence sandstone permeability.

24 At least two broad approaches are available for permeability prediction of sandstones: 1 )

25 physics-based models, such as the Carman-Kozeny equation and its derivatives, and 2)

26 empirical models, developed using statistical or machine learning (ML) tools that assume

27 no particular physical laws linking predictors and permeability. There are several physics-

28 based and empirical models; Dullien (2012) gives a good review of both model types. This

29 study included a hybrid approach that considers both the physical intuition encapsulated in

30 the Carman-Kozeny equation and data-centric models. The novelty of this work is that it

31 compares the results of the physics-only and physics plus data driven models.

32 Kozeny (1927) and Carman (1937) developed an equation linking permeability to three

33 factors: porosity, hydraulic tortuosity, and specific surface area. Porosity and permeability

34 are routinely measured during core analysis, but hydraulic tortuosity (as opposed to

35 electrical tortuosity) and specific surface area are rarely evaluated although some log-

36 derived quantities are surrogates for this. However, both tortuosity and specific surface

37 area arise from geologic processes that can be modeled and distributed throughout the

38 reservoir. Therefore, understanding the magnitude and effect of tortuosity and surface area 
39 can aid in building accurate permeability predictors and applying these predictions in

40 geomodels.

41 Panda and Lake (1995) developed a mathematical framework for estimating tortuosity and

42 specific surface area for real rocks that had undergone diagenesis. The framework, can

43 predict permeability from the intergranular porosity, the average grain diameter, the grain

44 size distribution, and the amounts and types of various cements.

45 Machine learning can be used to understand how useful tortuosity and specific surface area

46 are for predicting permeability. With advanced non-parametric ML (such as the gradient

47 boosting machine developed by Friedman, 2001), there is no requirement to assume $a$

48 priori a functional form between these variables and the predicted quantity. With the

49 recent derivation of a consistent feature attribution system for explaining tree-based

50 models (Lundberg et al., 2018), the functional form can be visualized after modeling; this

51 may help petrophysicists to understand the mechanisms controlling permeability.

52 In this study, we develop estimates for the permeability of the Garn Sandstone reservoir

53 (Ehrenberg, 1990), using the data from the 12 wells in that study. The Garn is a Middle

54 Jurassic formation in the North Sea, in the Haltenbanken area (Ehrenberg, 1990) that was

55 deposited in fluvial and near-shore marine environments (Gjelberg et al., 1987). It is

56 composed mostly of quartz grains and secondarily with feldspar (Ehrenberg, 1990). This

57 study compared different methods for calculating the tortuosity and specific surface area

58 from core description and found the most important determinants of permeability

59 predictors for this data. Our analysis shows that porosity best predicts permeability,

60 followed by the presence of pore bridging cement and then tortuosity. Given the physics- 
61 based model and advanced ML estimators, we propose a hybrid approach, combining the

62 best qualities of each method.

\section{Methods}

\section{Physical models}

65 Perhaps the best-known physics-based relationship to estimate permeability was

66 developed by Kozeny (1927) and later modified by Carman (1937). In its modern form, the

67 equation is written as

68

$$
k=\frac{\phi^{3}}{2 \tau(1-\phi)^{2} a^{2}}
$$

69 which, for simplicity, we will write as

$$
k=\frac{\phi_{C K}}{2 \tau a^{2}}
$$

71 where permeability is $k$, porosity is $\phi$, tortuosity is $\tau$, the specific surface area (wetted

72 area/volume) is $a$, and the Carman-Kozeny void fraction is $\phi_{C K}$. For an uncemented

73 sandstone, tortuosity can be calculated following the derivation in Appendix A, which

74 comes from Panda and Lake (1994). For a cemented sandstone (Appendix B), the tortuosity

75 changes because of cements blocking and forcing modification of the flow paths.

76 For monodisperse spheres, $a=6 / d$, where $d$ is the sphere diameter. For uncemented

77 spheres of more than one size, $a$ can be estimated from the particle size distribution

78 (sorting) (Panda and Lake, 1994). After cementation, the cement distribution is a further 
79 control on how the surface area changes. Some cements will coat the pores walls, slightly

80 decreasing the specific surface area. Other cements will line or bridge the pores,

81 moderately to greatly increasing the specific surface area.

82 A different model is based on the idea that pore throat sizes are an important variable in

83 permeability models. This hypothesis is implicit in the Winland-style relations that follow

84 the form

$$
\ln r=A \ln k-B \ln \phi+C
$$

86 where $r$ is the pore throat radius (see Kolodzie, 1980; Di and Jensen, 2015).

87 Doyen (1988) formalized this approach, applying effective medium theory to explain

88 permeability with the equation

89

90

$$
k=\frac{\phi}{8 \tau} \frac{r_{e f f}^{4}}{\left\langle r_{t}^{2}\right\rangle}
$$

91 where $r_{e f f}$ is the effective pore throat radius and $\left\langle r_{t}^{2}\right\rangle$ is the spatial average of the square of

92 the pore channel radii. This result is remarkably similar to the Carman-Kozeny equation,

93 except that the dependency on specific surface area has been replaced with a dependency

94 on the pore throat radii.

95 As a practical consideration, the pore throat radius might be more impacted by cements

96 that coat the walls than cements that bridge the pores. However, the opposite is true for the

97 specific surface area (Scheidegger, 1960). 


\section{Data-driven models}

99 Empirical models have long been important in reservoir engineering (see Frick, 1962 for

100 numerous examples). These models, such as Winland's equation (Kolodzie, 1980), seek out

101 relationships between predictor variables (independent variables) and responses

102 (dependent variables - here, permeability). In the last two decades, advances in applied

103 statistics and computing power have created new approaches for developing empirical

104 relationships. This has spawned the field of data analytics and the attendant study of ML.

105 The data analytics approach is as follows:

106 1. collect and clean data

107 2. propose physics-based predictor variables

108 3. perform exploratory analysis

109 4. build machine learning models on a subset of the data (training data)

110 5. evaluate the machine learning models on new data (testing data)

111 6. interpret model results.

112 We apply the above workflow to data from Ehrenberg (1990). This dataset has a large

113 range of permeability and porosity, cement proportions are measured, and it requires only

114 minimal cleaning. However, the data lacks many of the variables in the Carman-Kozeny

115 equation. Therefore, we performed feature engineering to derive these variables from

116 Ehrenberg's measurements. Among the variables the data did not have were the mean

117 particle size, the coefficient of variation of the particle size, and the skewness of the particle

118 size distribution. These variables were derived through the procedure given in Appendix C. 
119 During exploratory data analysis, we plot the distributions of predictor and response

120 variables and make cross-plots between variable pairs to identify predictor variables with

121 strong co-linearity and with strong correlation to the response variable. For the Garn

122 sandstone, the predictor variables include the porosity, the Carman-Kozeny void fraction,

123 the Carman-Kozeny predictions of permeability, and the volume fractions of pore-filling

124 and pore-bridging cement present.

125 Ehrenberg (1990) estimated porosity two ways: Helium porosimetry, and point counting

126 the intergranular macroporosity of thin sections. These measurements are highly

127 correlated, so including both in the regression model could cause overfitting and

128 overestimate the influence of porosity on the permeability (feature importance would be

129 split between the two porosity measures). Therefore, we chose to use a single porosity

130 estimate. Exploratory data analysis showed that intergranular macroporosity was a better

131 predictor of permeability than Helium porosity, and we chose it for the model.

132 This study includes two approaches to building the models: multiple linear regression and

133 gradient boosting regression (Friedman, 2001). Multiple linear regressions are common,

134 easily interpretable, and robust to overfitting. These regressions also make several

135 assumptions that are often violated in real data sets, including a linear model relating

136 predictors and response variables, Gaussian distributions, and homoscedastic residuals.

137 Gradient boosting regressors make fewer assumptions about the distributions of the input

138 data and the character of the relationship between predictor variables and the response,

139 but their results are difficult to interpret and prone to overfitting. To illustrate the benefits

140 and drawbacks of these approaches, we use both methods and compare the results. 
141 Through careful feature selection and pre-processing, we limited the degree to which the

142 assumptions in linear regression are violated. As aforementioned, one of those steps is

143 removing highly correlated predictor variables. In addition, we log-transformed the

144 predictor variables and permeability, which reduces non-normality of the variables'

145 distributions. Log-transformation also makes the correlations between variables more

146 linear. Using the Box-Cox (1964) transformation did not significantly improve the results,

147 but it can be effective in some cases, as shown by Jensen et al. (1987).)

148 We evaluated the models through calculating the model explained variance $\left(R^{2}\right)$, mean

149 absolute error (MAE), and root-mean squared error (RMSE). The equations for these

150 measures are as follows

151

152

$$
\begin{aligned}
R^{2} & =\frac{1}{n-1} \sum_{i}^{n} \frac{y_{i}-\bar{y}}{\sigma_{y}} \frac{y_{i}^{\prime}-\overline{y^{\prime}}}{\sigma_{y^{\prime}}} \\
\operatorname{RMSE} & =\left(\frac{1}{n} \sum_{i}^{n}\left(y_{i}-y_{i}{ }^{\prime}\right)^{2}\right)^{1 / 2} \\
\text { MAE } & =\frac{1}{n} \sum_{i}^{n}\left|y_{i}-y_{i}{ }^{\prime}\right|
\end{aligned}
$$

153 where $n$ is the sample size $i$ represents the sample number, $y$ is the actual value, $y^{\prime}$ is the

154 predicted value, a bar over a quantity is the sample mean of that quantity, and $\sigma$ is the

155 sample standard deviation of a quantity.

156 Hyperparameters for the gradient boosting regressor were selected through cross-

157 validation. During cross-validation, candidate models are fed data on seven of the eight

158 wells in the training data, then scored based on which minimizes the RMSE predicting the 
159 excluded well. This is iterated through each well and a gamut of hyperparameters. Through

160 this procedure, we maximize the model effectiveness while reducing overfitting by

161 minimizing the validation RMSE on held-out data (four wells in the testing data).

162 In order to determine whether predictor variables contributed to the result, we used a non-

163 parametric approach. This approach is called Permutation Feature Importance (Fisher, et

164 al., 2018), and estimates the importance of a predictor variable based on how much the

165 model error increases after that variable is permutated (randomly shuffled).

166 Linear models can be interpreted simply through examining the weight assigned to each

167 predictor (feature). Gradient boosting methods require a different approach. SHapley

168 Additive exPlanations (SHAP values) offer a way to explain how each predictor variable

169 contributed to each prediction (Lundberg and Lee, 2017). The idea behind Shapley values

170 is to determine how much each input affects the output for each individual prediction. To

171 do this, SHAP values use an idea borrowed from cooperative game theory (Shapley, 1953),

172 where the actors work together as a team to achieve a result, leading to a pay-out

173 proportional to how much each actor contributed to the final result. We use an exact

174 solution for SHAP values (Lundberg and Lee, 2017) that has been implemented in the

175 XGBoost library (Chen and Guestrin, 2016). 


\section{Results and Discussion}

\section{Exploratory analysis}

178 First, we examined the distributions for porosity, permeability, Carman-Kozeny void

179 fraction, and the proportion of various cements (Fig. 1). The permeability, porosity-and

180 therefore Carman-Kozeny void fraction-distributions follow a bi-modal distribution. The

181 permeability histogram is the most clearly bi-modal (modes of approximately 0.8 and 90

$182 \mathrm{md})$ of the three parameters, but a minor mode also exists in the porosity histograms $(\log \phi$

183 modes at approximately 1.8 and $6 \mathrm{pu}$ ). Multimodal distributions are common in subsurface

184 data and can be indicative of multiple facies (Jensen et al., 2000). An appropriate treatment

185 of bi-modal data is to analyze each mode separately, splitting the analysis into high

186 porosity and low porosity assessments.

187 Therefore, when we performed regressions on the data, we treated each mode separately,

188 rather than regressing across the entirety of the data. The data was split into two classes:

189 samples where the interparticle macro-porosity is greater than $2.3 \%$ (high) or less than or

190 equal to 2.3\% (low). The cutoff was selected through using Gaussian Mixture Modeling

191 (Fraley and Raftery, 2002) to separate the modes.

192 There are 163 points in the high porosity training set, 41 points in the high porosity testing

193 set, 48 points in the low porosity training set, and 20 points in the low porosity testing set. 

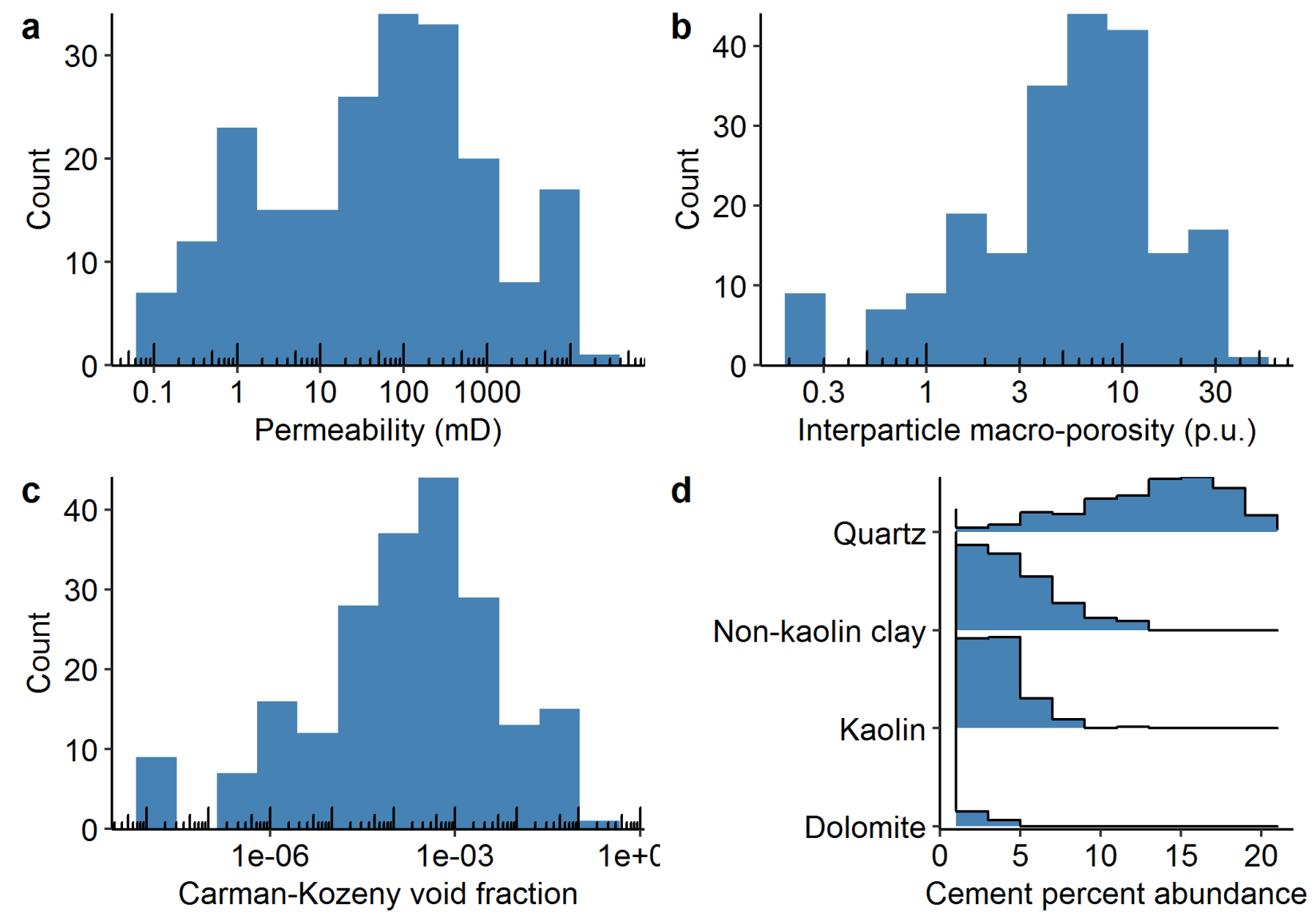

195 Figure 1. Histograms for the distributions of a) Klinkenberg-corrected absolute

196 permeability b) interparticle macro-porosity from point-counting c) Carman-Kozeny void

197 fraction from macro-porosity d) Percent abundances (total area fraction) of cement. The

198 permeability and porosity, both log-transformed, follow bimodal distributions. Quartz is

199 the most abundant cement, followed by non-kaolin clay (smectite and illite).

200 Next, we cross-plotted permeability against several individual predictors (Fig. 2): Carman-

201 Kozeny void fraction, tortuosity, pre-cementation specific surface area, and fraction of

202 pore-bridging and pore-filling cement. Pore-filling cement includes quartz, kaolin clay, and 
203 dolomite, and pore-bridging cement is non-kaolin clay.
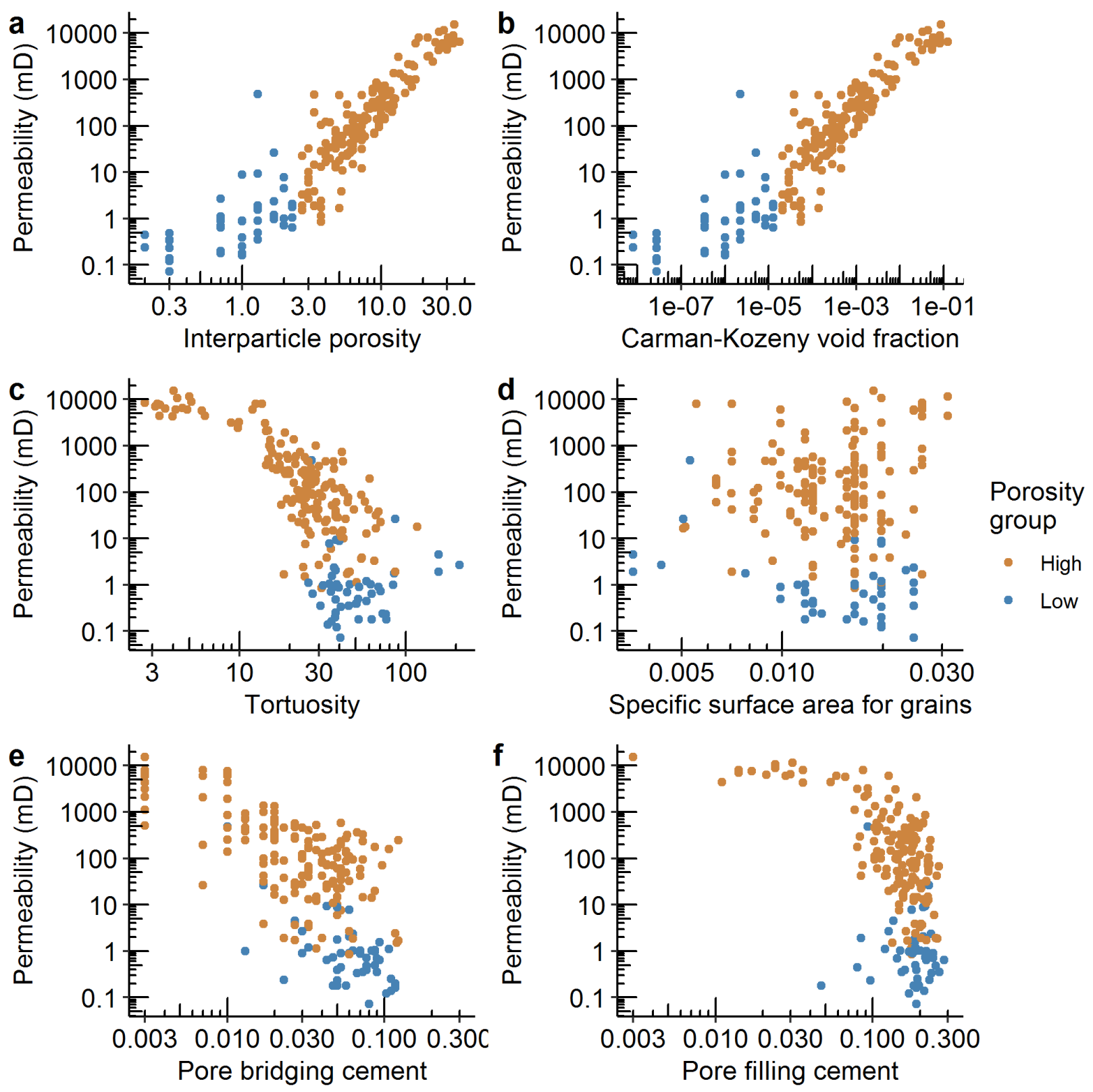

205 Figure 2. Cross-plots between permeability and several predictor variables. These variables

206 include a) the interparticle macro-porosity b) the Carman-Kozeny void fraction, c)

207 tortuosity as calculated in Panda and Lake (1995), d) specific surface area in reciprocal

208 square microns for the grains (pre-cementation), e) fraction of pore-bridging cement f) 
209 fraction of pore-filling cement. The color indicates whether the sample has greater than 2.3

210 percent porosity (orange) or not (blue).

211 To assign values to the correspondence between the predictor variables and permeability,

212 we calculated the Pearson's $r$ and Kendall tau values (Table 1). Both statistics measure the

213 degree of association between the variables and have values between -1 and 1 . Pearson's

214 statistic is a measure of linear correlation and based on the data values; Kendall's statistic

215 is based on the ranks of the data values. More details can be found in many statistics texts,

216 including Miller (1986).

217 Table 1. Pearson $r$ and Kendall tau values for correlation between log-transformed

218 predictor variables and log permeability. The data is split between modes of the porosity

219 distribution, based on whether or not the porosity is greater than 2.3. Tortuosity is

220 calculated after taking cementation into account; specific surface area is calculated without

221 including cementation - making the presence of pore-bridging and pore-filling cements

222 into proxies for specific surface area.

\begin{tabular}{lrrrrrr} 
& & Carman- & & Specific & Pore- & Pore- \\
Porosity & & Kozeny void & & surface & bridging & filling \\
group & Correlation & fraction & Tortuosity & area & cement & cement \\
\hline High & Pearson r & 0.90 & -0.77 & 0.18 & -0.71 & -0.63 \\
High & Kendall tau & 0.73 & -0.57 & 0.11 & -0.46 & -0.40 \\
Low & Pearson r & 0.48 & 0.06 & -0.42 & -0.59 & -0.09 \\
Low & Kendall tau & 0.44 & 0.02 & -0.15 & -0.31 & -0.06
\end{tabular}


223 The two correlation measures show similar values within each porosity group, however

224 they take on different values between the porosity groups, with less correlation at low

225 porosity. Porosity is the most strongly correlated with permeability, with cements next,

226 and tortuosity and specific surface area having the weakest correlations.

\section{Model results}

228 This study tested the accuracies and correlations between the physics-based and

229 regression-based models and the measured permeability. The three physics-based models

230 of increasing complexity are:

231 1. Classic Carman-Kozeny model with no compaction or cementation effects

232 2. Carman-Kozeny model with the effect of compaction on the grain size distribution

233 3. Carman-Kozeny including compaction and cement's effect on tortuosity

234 The results from these models of increasing complexity are shown in Fig. 3. 


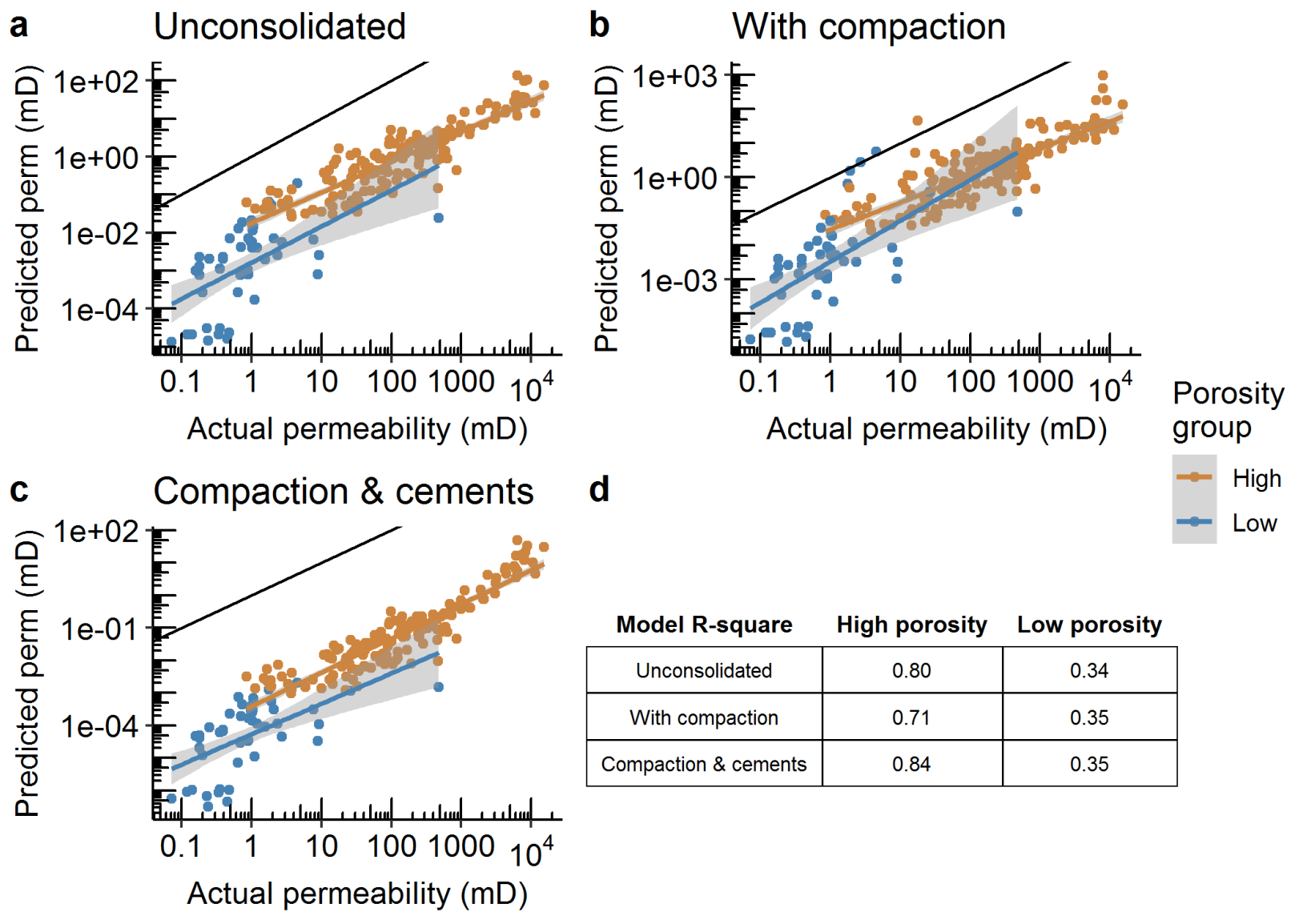

236 Figure 3. Comparisons of physics-based models to measured permeability. The black line

237 indicates perfect agreement. The colored lines are least-squares best fits. Shading indicates

$23895 \%$ uncertainty in the best fit line. a) Uses the Carman-Kozeny void fraction and the initial

239 tortuosity and specific surface area expected from an uncompacted particle assemblage of

240 the measured porosity and grain size. b) Considers compaction with the Panda-Lake

241 (1994) model. c) Considers the impact of compaction and the effect of cementation on the

242 tortuosity, following the Panda-Lake (1995) model. d) R² for the log-permeability predicted

243 by these models compared to observed in the core.

244 Including compaction and cementation modestly improves the Carman-Kozeny model $\mathrm{R}^{2}$

245 by 0.05 for the high porosity sandstone, but weakly for low porosity samples (Fig 3d). High 
246 porosity samples are better predicted than low porosity samples. All sample permeabilities

247 are significantly underpredicted by approximately two to three orders of magnitude by the 248 physics-based models, which have no fitting parameters.

249 In addition to the three physics-based models, we tested two physics-inspired, regression-

250 based models (Fig. 4):

251 4. A linear model using a Winland-style equation of the form

$$
\ln k \propto \ln \phi_{C K}+\ln a_{u}+\ln \tau_{e}+\ln P_{b}+\ln P_{f},
$$

254 5. A gradient boosting model using the same predictor variables, but assuming no

255 particular functional form between the variables and permeability 

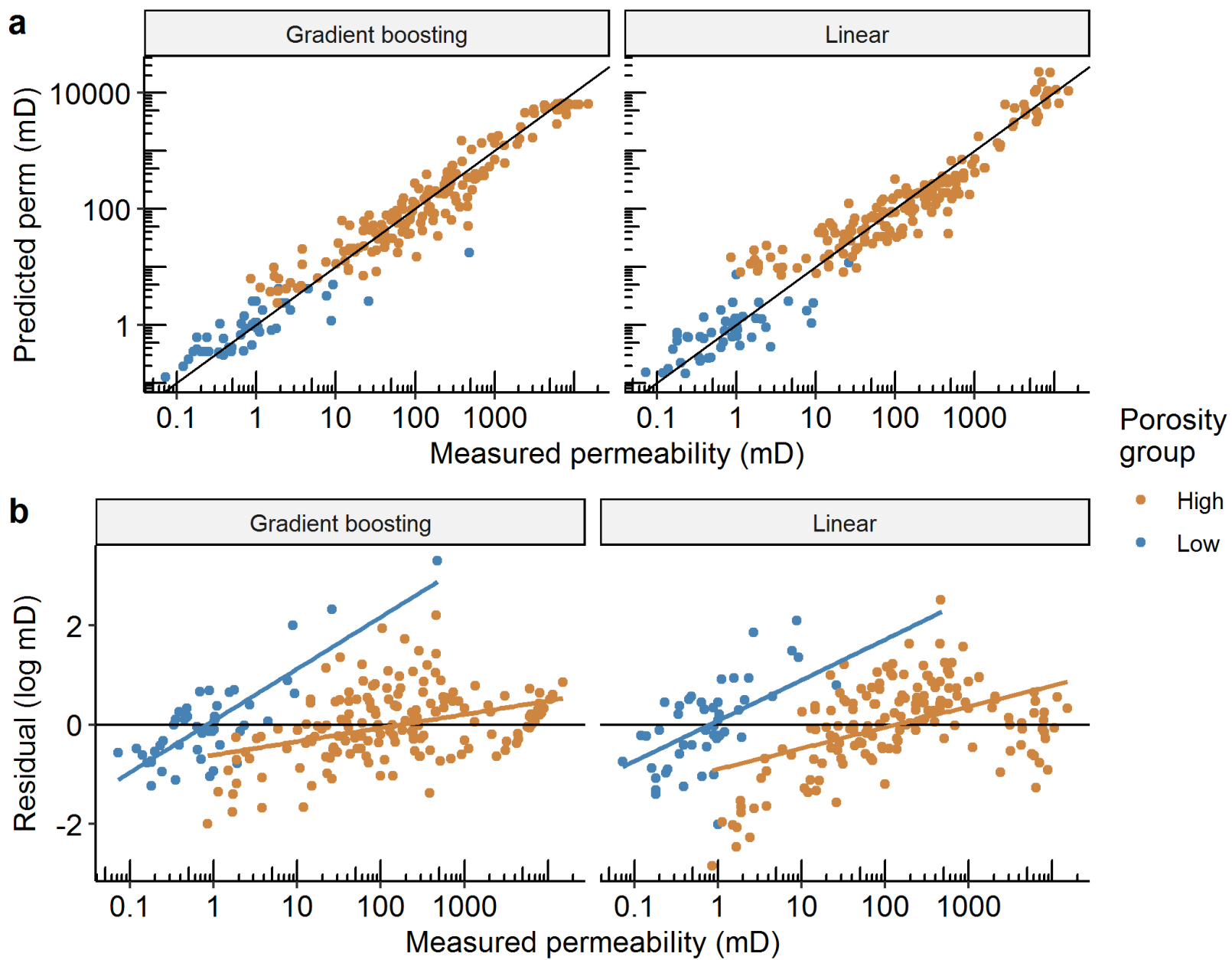

- High

- Low

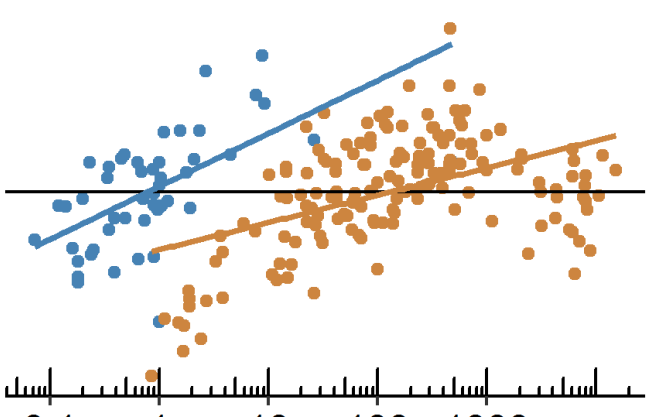

257 Figure 4. a) Predicted versus measured permeability using the linear and gradient boosting

258 models. b) Residuals in the predictions for the linear and gradient boosting models. Color

259 indicates whether the sample is in the high (greater than 2.3\%) or low porosity group.

260 Lines indicate the trends in the residual.

261 The XGBoost hyperparameters that best match permeability for low porosity rock are 520

262 trees, a learning rate of 0.02 , no minimum loss reduction (gamma), a max tree depth of 1 ,

2630.78 of the columns sampled by each tree, and a minimum child weight of 7 samples. For

264 the high porosity rock, they were 550 trees, a learning rate of 0.017 , a maximum tree depth 
265 of 2, a minimum loss reduction of $0.94,0.69$ of the columns sampled by each tree, a

266 minimum child weight of 2 , and a subsample ratio of 0.23 for the training instances.

267 The linear model Elastic net hyperparameters that best match permeability for low

268 porosity samples cause no regularization. For high porosity samples, the hyperparameters

269 are a regularization constant of 0.15 and an alpha of 0.02 , indicating primarily ridge style

270 regression.

271 As perhaps best shown by the residuals and best fit lines (Fig. 4b), neither model is

272 explaining all the permeability variation with the chosen predictors and models. That is to

273 say, there is a functional relationship between the residual values of the prediction and the

274 value of the permeability. Fig. 4a gradient boosting shows no predictions above $5220 \mathrm{md}$, a

275 result of the minimum number of points allowed in each split of the gradient boosting

276 trees. Fig. 4b shows that residuals follow a quadratic function at high porosity, indicating a

277 higher-order (than linear) relationship between one or more of the predictors and

278 permeability.

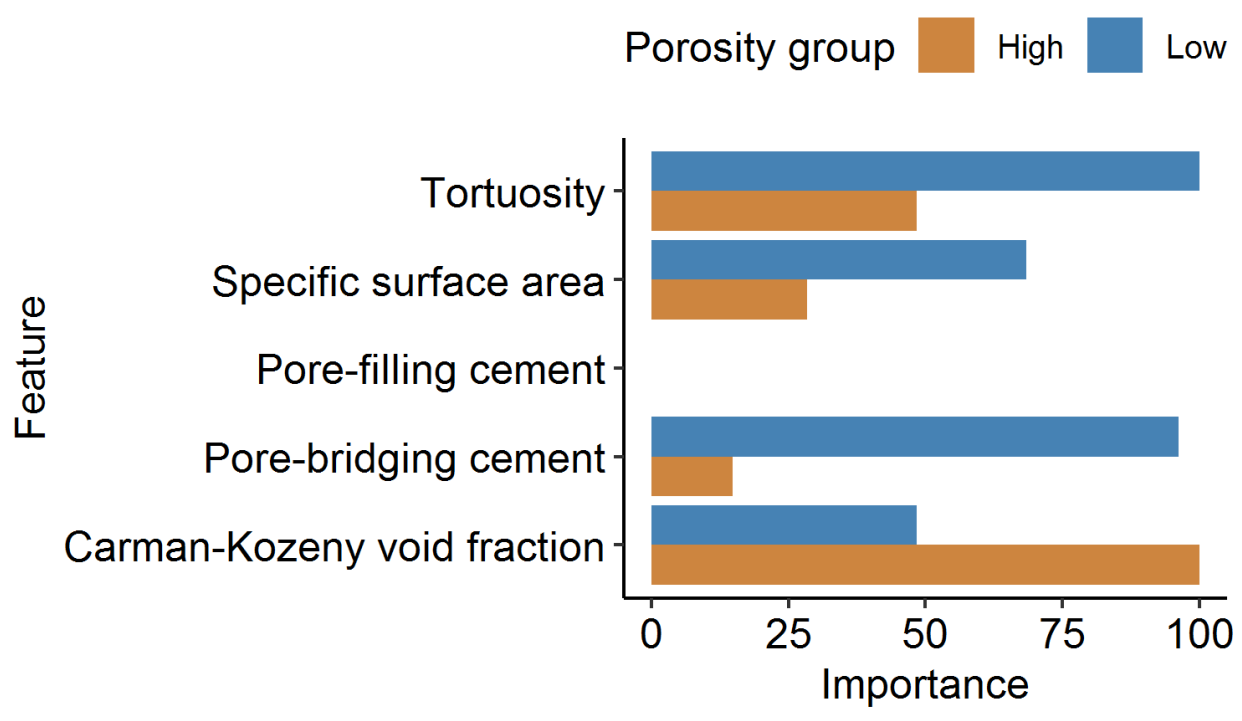


280 Figure 5. Feature importance for the linear model. Color indicates whether the model was 281 trained on high (greater than 2.3\%) or low porosity samples. No bar indicates that the 282 regularization procedure caused the weight for that feature to reach zero.

283 The linear model shows different features are important for high versus low porosity

284 samples (Fig. 5). Carman-Kozeny void fraction is the most important factor for high

285 porosity rock, followed by tortuosity and specific surface area. For low porosity samples,

286 tortuosity and the fraction of pore-bridging cement are the most important features. In

287 neither group is the fraction of pore-filling cement an important feature, both models

288 assign it zero weight (i.e., it does not directly influence permeability).

Porosity group - High • Low
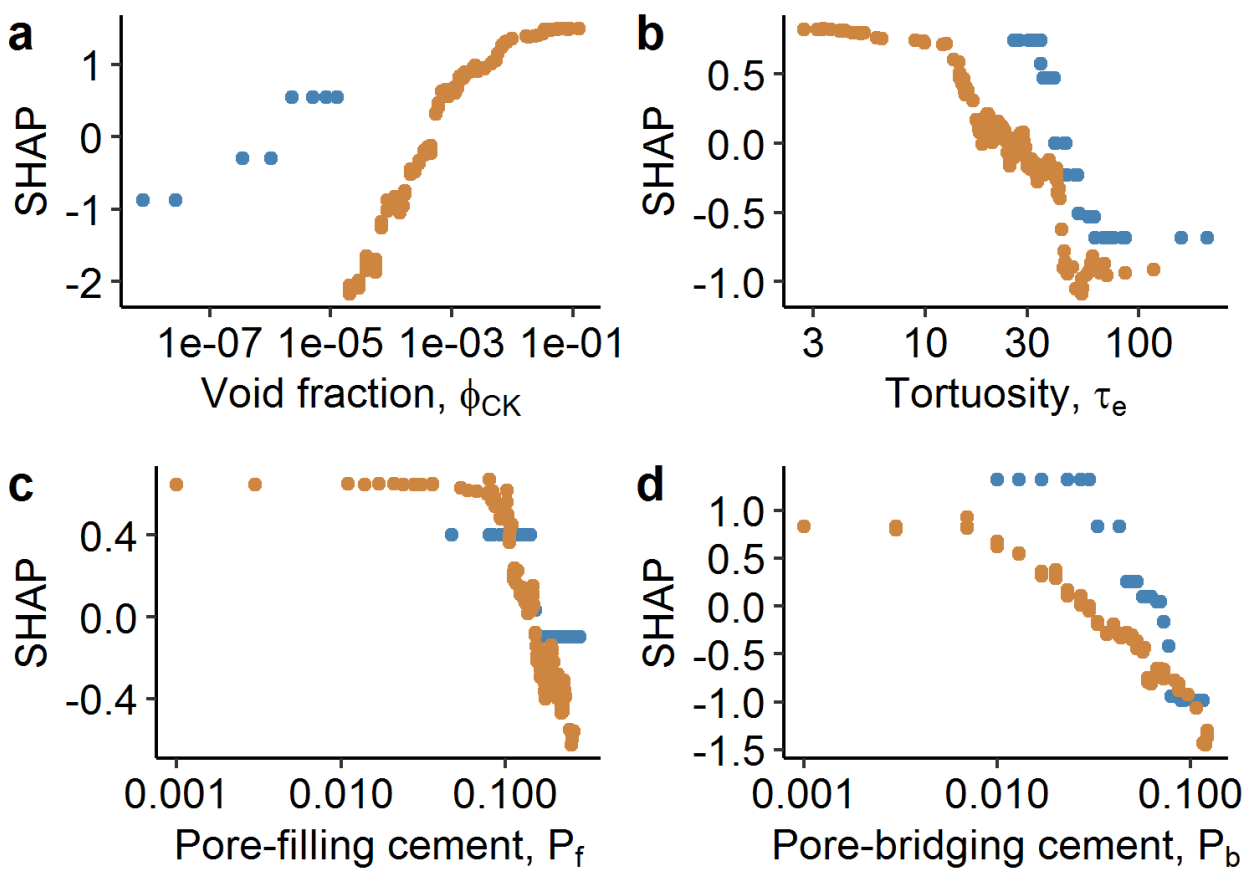

290 Figure 6. Feature importance for the gradient boosting model, using SHapely Additive 291 exPlananations (SHAP). SHAP values use game theory to explain how much each element 
292 contributes to each prediction from the gradient boosting model. Orange dots show high

293 porosity samples, while blue samples indicate low porosity samples. The SHAP values are

294 for the following features: a) Carman-Kozeny void fraction b) Tortuosity c) volume fraction

295 of pore-filling cement d) volume fraction of pore-bridging cement.

296 For the gradient boosting model (Fig. 6), each dot represents the importance for a

297 particular sample. A zero SHAP value indicates no influence of the chosen predictor on the

298 permeability for that sample. The largest influence on permeability comes from void

299 fraction for high porosity samples, and the fraction of pore-bridging cement for the low

300 porosity samples. Tortuosity and the fraction of pore-filling cement are of secondary

301 importance, and the specific surface area (before cementation) is least important.

302 The SHAP values for pore filling cement concentration follow a sharply sigmoidal shape,

303 implying a transition point around $10 \%$ cementation. Other features show more samples in

304 their linear trend, with the effects of extreme points leveling off because of limited data.

305 Table 2. Measures of model fitness for the gradient boosting, linear, and advanced Carman-

306 Kozeny models on the high porosity and low porosity groups for the training and testing 307 data.

\section{Porosity}

\begin{tabular}{llllll} 
Model & group & Data & RMSE & MAE & $\mathrm{R}^{2}$ \\
\hline Gradient & Low & Train & 0.85 & 0.59 & 0.74
\end{tabular}

boosting 


\begin{tabular}{|c|c|c|c|c|c|}
\hline $\begin{array}{l}\text { Gradient } \\
\text { boosting }\end{array}$ & Low & Test & 1.31 & 1.07 & 0.52 \\
\hline Gradient & High & Train & 0.71 & 0.55 & 0.90 \\
\hline boosting & & & & & \\
\hline Gradient & High & Test & 0.97 & 0.72 & 0.83 \\
\hline boosting & & & & & \\
\hline Linear & Low & Train & 0.85 & 0.68 & 0.69 \\
\hline Linear & Low & Test & 1.74 & 1.35 & 0.49 \\
\hline Linear & High & Train & 1.64 & 1.33 & 0.69 \\
\hline Linear & High & Test & 1.73 & 1.37 & 0.67 \\
\hline Carman-Kozeny & Low & Train & 10.00 & 9.81 & 0.35 \\
\hline Carman-Kozeny & Low & Test & 12.13 & 11.99 & 0.44 \\
\hline Carman-Kozeny & High & Train & 7.70 & 7.63 & 0.84 \\
\hline Carman-Kozeny & High & Test & 8.17 & 8.08 & 0 \\
\hline
\end{tabular}

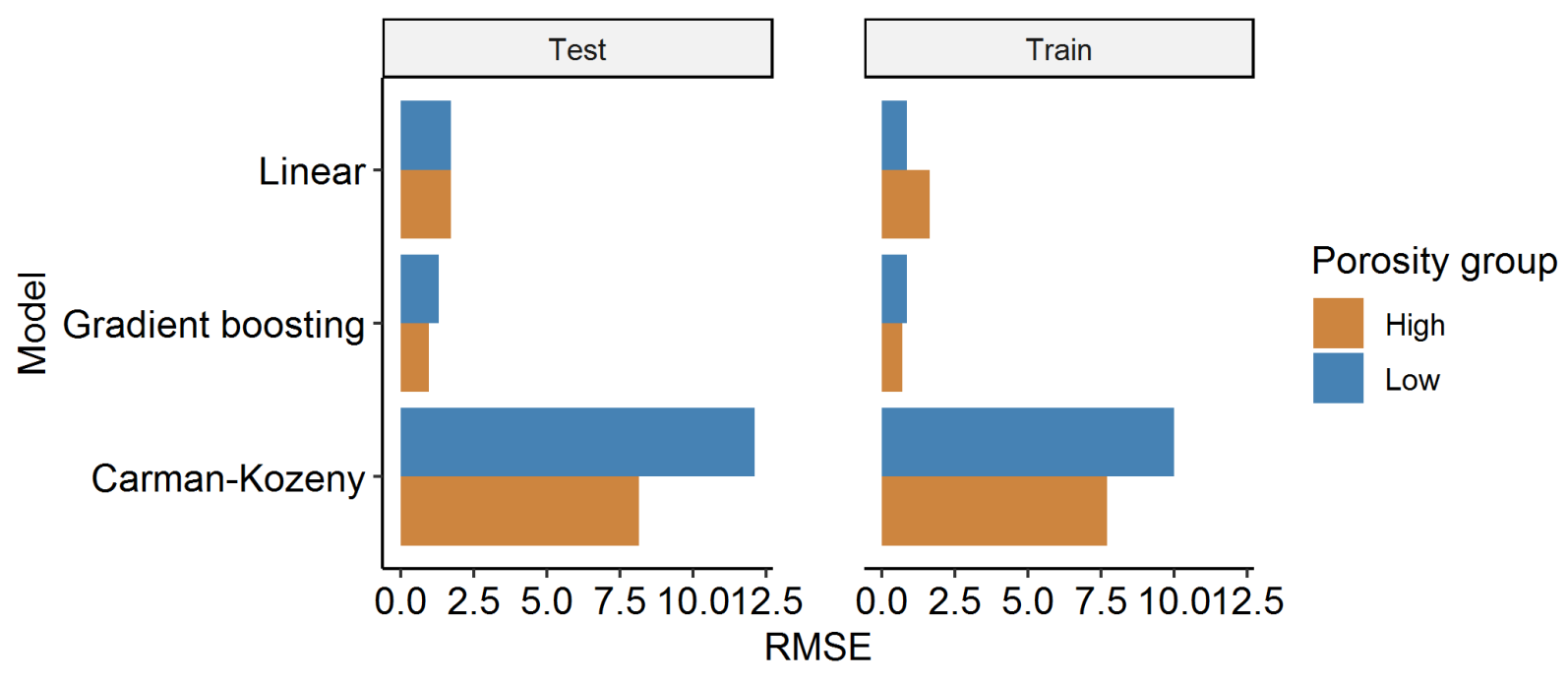


309 Figure 7. Comparison of the root-mean-squared errors for each model. The testing errors

310 are on the right, and the training errors are on the left. The gradient boosting model shows

311 the smallest errors, followed by the linear Winland-style model. The advanced Carman-

312 Kozeny model has the largest errors. Color indicates whether the porosity is above or

313 below the cutoff.

314 The model metrics (Table 2) indicate that the gradient boosting method leads to smaller

315 residuals and a higher $\mathrm{R}^{2}$ than the linear model. This is clearly illustrated in the RMSE plot

316 in Figure 7. For most cases, the models work better on cross-validation training data than

317 testing data. For comparison, a porosity-only log-linear model has $\mathrm{R}^{2}=0.81$ for high

318 porosity, 0.23 for low porosity. The gradient boosting model has better explanatory value

319 than porosity alone, while the linear model has roughly the same explanatory value. Both

320 models outperform the porosity-only model at low porosity. They also significantly

321 outperform the physics-based models at low porosity.

322 Paired t-tests were performed on the residuals for each model for the training and testing

323 data. The null hypothesis was accepted that gradient boosting and linear models did not

324 differ statistically significantly on the low porosity data. At high porosity, the alternative

325 hypothesis was accepted that gradient boosting model performed better in both RMSE and

326 MAE, for both the training and testing data sets, with p-values less than 0.01 .

\section{Discussion}

328 We have presented several methods for estimating permeability from thin section data for

329 sandstone samples. First, we used several physics-based models of increasing complexity. 
330 Then, we built hybrid data-driven models with physical parameters as inputs. The data-

331 driven models performed better than the purely physics-based models.

332 A key step in this analysis is splitting the data into two parts, each containing one mode of

333 porosity. Why have we done this? During exploratory analysis, we saw that the

334 permeability distribution was bi-modal, and the porosity distribution did not match either

335 a normal or a log-normal distribution. Multi-modal permeability distributions are a

336 common problem in permeability modeling (see, e.g. Clarke, 1979; Dutton and Willis, 1998;

337 and Jensen et al., 2000). One approach for treating multiple modes is to split the

338 distribution by mode and analyze each separately. This approach is particularly useful for

339 reservoirs, where identifying the causes for high permeability zones is important, and the

340 magnitude of low permeability zones may be less important. The splits can be selected

341 through visual inspection, Gaussian Mixture Models (Fraley and Raftery, 2002), or k-means

342 clustering (Likas et al., 2003). Gaussian Mixture Models were used in this study.

343 The next step of the exploratory analysis is summarized in Table 1. Consistent with many

344 other studies (e.g., Amyx et al., 1960; Slatt, 2006; Doveton, 2014; Baker et al., 2015), we see

345 that porosity has a strong correlation with permeability for the larger-porosity data. There

346 is, however, little to no correlation for low permeability rock, similar to patterns observed

347 elsewhere (e.g., Broger et al., 1997, their Fig. 10; Wendt et al., 1986, their Figs. 2 and 7). In

348 fact, no single parameter correlates strongly with permeability for the low-porosity

349 samples. The Pearson and Kendall correlations are informative, but determining true

350 feature importances requires interrogating a regression model. 
351 Physics-based models based on successively more complex modifications of the Carman-

352 Kozeny equation were tested on the data. We found that including the effect of compaction

353 on the flow properties was not sufficient to improve the model without including

354 cementation. This is in contrast to the findings of Panda and Lake (1994), but consistent

355 with their later work (Panda and Lake, 1995), which included cementation.

356 Two ML-based models were trained and tested on the data. The Winland-style linear model

357 was the less accurate of the two, but it still provided insights into the relative importance of

358 different physical effects on permeability. The gradient boosting model was more accurate

359 overall and showed a nonlinear effect coming from cementation. However, in a relatively

360 low data environment it loses some resolution in the predictions at the extremes of high

361 and low permeability (see Fig 4a, the top 10 permeability points).

362 The benefits of using the linear model were 1) The model is relatively simple with few

363 parameters to evaluate; 2) the permeabilities above $5000 \mathrm{md}$ were better predicted than

364 with the gradient boosting model. The gradient boosting model, however, could be used

365 with SHAP evaluations to identify control strengths for each sample. This option could be

366 quite useful in other cases if geological information were also available. For example, one

367 might look for changes in the strengths of the predictor variables according to the facies

368 from which the sample was taken.

369 All of the models tested performed worse at predicting permeability at low porosity. This is

370 likely because of the higher tortuosity and specific surface areas, more cementation, and

371 smaller pore throats at this porosity range. Alternatively, we might have failed to measure

372 an important permeability predictor. 
373 The physics-based model performed worse than the data analysis based models by most

374 metrics, but this does not mean it is without use. Although the absolute values of

375 permeability are severely underestimated, the $\mathrm{R}^{2}$ show good agreement at high

376 permeability, better than the Winland model (Table 2). Considering that the Winland

377 model uses the Carman-Kozeny equation components as its input, this could indicate

378 overfitting for that particular regression. Overfitting is endemic to all empirical models, and

379 although it can be minimized through cross-validation and test-training splits, it cannot be

380 removed without introducing bias to the results. See Breiman (1996) for discussion on bias

381 and variance in data analysis.

382 There has been healthy debate on whether Doyen's (1988) pore throat size based approach

383 or Panda and Lake's (1995) specific surface area approach tell us more about the

384 permeability of sandstones. After building and interpreting two machine learning models,

385 this study can now shed some light on the question.

386 The feature importances from the logarithmic regression provide evidence that pore throat

387 size is more important than specific surface area in determining permeability. On the other

388 hand, the degree of pore-filling cement present is not important. This recommends

389 measuring pore throat sizes over determining specific surface area.

390 From the gradient boosting model, we see that specific surface area is less important than

391 void fraction, tortuosity, and the degree of cementation. However, this measure of specific

392 surface area does not include the cementation effect because Panda and Lake did not

393 provide values for calculating surface area from the amount of pore-filling and pore- 
394 bridging cement. We see from the SHAP values (Figure 6) that this could be a strong effect 395 following a sigmoidal functional form.

396 This is, to our knowledge, the first study using SHAP values to interpret machine learning 397 results in predicting permeability. Erofeev, et al (2019) used gradient boosting regressors

398 to predict permeability, but only used F scores to find the most important parameters and

399 did not attempt to determine the functional dependence for each feature. Other studies that

400 have used machine learning to predict permeability but not interpreted their models,

401 include Huang et al. (1996) and Rezaee et al. (2006). Al-Mudhafar (2019) used a Bayesian

402 approach to determine which predictors were influential for predicting permeability but 403 used linear models.

404 The SHAP values for pore-filling and pore-bridging cement indicate that pore-bridging 405 cement is more important for determining permeability, which is consistent with either a 406 surface area or pore throat-centric paradigm. However, for all cements, there appears to be 407 a threshold around $10 \%$ volume fraction, after which permeability drops drastically. This 408 could indicate that, while specific surface area and pore throat radius are both good 409 explanatory variables for interpreting permeability, at around $10 \%$ cementation, pores and 410 pore throats are blocked, and this is the dominant effect on permeability. From another 411 perspective, $10 \%$ cements could be interpreted as a percolation threshold. This value is 412 less than the threshold values suggested by Korvin (1992) (0.25 to 0.5) but within the 413 range of values calculated by Deutsch (1989) (0.1 to 0.5). 


\section{Conclusions}

415 We used a sandstone dataset to test several models for predicting permeability in the

416 presence of cementation. We found the following:

417 1. Machine learning provides better data correlation than even advanced Carman-

$418 \quad$ Kozeny models.

419 2. Gradient boosting can improve upon linearized regression, and helps to identify 420 nonlinear effects coming from cementation.

421 3. As a first step analysis, porosity is a remarkably good predictor of permeability at

422 porosities greater than $2.3 \%$, after it has been transformed to Carman-Kozeny void 423 fraction.

424 4. To improve upon porosity-only predictions in sandstones using thin section analysis, 425 pore-bridging cement amounts should also be evaluated.

426 5. For the Garn sandstone, the importance of variables is as follows:

427 - High porosity: porosity, cements, tortuosity, and specific surface

428 - Low porosity: pore-bridging cement, porosity, tortuosity, pore-filling cement

\section{Acknowledgements}

430 We are grateful to Stephen Ehrenbreg for the kind donation of his core analysis of the Garn

431 formation. We thank Ian Duncan and William Ambrose for valuable discussions during the 432 preparation of this manuscript and Behzad Ghanbarian for suggestions regarding

433 theoretical calculations of tortuosity. This study was funded in part by the US Department 
434 of Energy (DOE) grant FE0024375 (PI: Ian J. Duncan) and in part by the State of Texas

435 Advanced Resource Recovery Program (PI: William A. Ambrose). Statistical analysis was

436 performed in the R language (R Core Team, 2014). Plots were generated using the GGPlot2

437 package (Wickham, 2009). Larry W. Lake holds the Shahid and Sharon Chair at the

438 Hildebrand Department of Petroleum and Geosystems Engineering.

\section{References}

440 Ahmadi, M.M., S. Mohammadi, and A. Nemati Hayati, 2011. Analytical derivation of

441 tortuosity and permeability of monosized spheres: A volume averaging approach. Phys.

442 Rev. E 83:026312. doi:10.1103/PhysRevE.83.026312

443 Al-Mudhafar, W.J., 2019. Bayesian and LASSO regressions for comparative permeability

444 modeling of sandstone reservoirs. Natural Resources Research, 28(1), pp.47-62.

445 Amyx, J. W., Bass, D. M., and Whiting, R. L., 1960, Petroleum Reservoir Engineering, McGraw446 Hill.

447 Baker, R. O., Yarranton, H. W., and Jensen, J. L., 2015, Practical Reservoir Engineering and 448 Reservoir Characterization, Gulf Prof. Pub.

449 Beard, D.C. and Weyl, P.K., 1973. Influence of texture on porosity and permeability of

450 unconsolidated sand. AAPG Bulletin, 57(2), pp.349-369.

451 Box, G. E. P., Cox, D.R., 1964. An analysis of transformation revisited, rebutted, Journal of 452 the American Statistical Association, 77, pp. 209-210.

453 Breiman, L., 1996. Bias, variance, and arcing classifiers. Tech. Rep. 460, Statistics

454 Department, University of California, Berkeley, CA, USA. 
455 Broger, E. J. K., Syhlonyk, G. E., and Zaitlin, B. A., 1997. Glauconite Sandstone exploration: A

456 case study from the Lake Newell Project, southern Alberta. In Petroleum Geology of the

457 Cretaceous Mannville Group, Western Canada, S. G. Pemberton and D. P. James (eds.), CSPG

458 Memoir 18, pp 140-168.

459 Carman, P.C., 1937. Fluid flow through granular beds. Trans. Inst. Chem. Eng., 15, pp.150-

460166.

461 Chen, T. and Guestrin, C., 2016, August. Xgboost: A scalable tree boosting system. In

462 Proceedings of the 22nd ACM SIGKDD international conference on knowledge discovery and

463 data mining (pp. 785-794).

464 Comisky, J. T., Newsham, K. E., Rushing, J. A., and Blasingame, T. A., 2007. A comparative

465 study of capillary-pressure-based empirical models for estimating absolute permeability in

466 tight gas sands. SPE conference paper SPE 110050 presented at the SPE Ann. Tech. Conf.

467 and Exhib., Anaheim CA 11-14 Nov., 18p.

468 Clarke, R.H., 1979. Reservoir properties of conglomerates and conglomeratic sandstones.

469 AAPG Bulletin, 63(5), pp.799-803.

470 Di, J. and Jensen, J.L., 2015. A closer look at pore throat size estimators for tight gas

471 formations. Journal of Natural Gas Science and Engineering, 27, pp.1252-1260.

472 Deutsch, C., 1989, Calculating effective absolute permeability in sandstone/shale

473 sequences. SPE Form. Eval., vol. 4(3), pp343-348.

474 Doveton, J. H., 2014, Principles of Mathematical Petrophysics, Oxford University Press.

475 Doyen, P.M., 1988. Permeability, conductivity, and pore geometry of sandstone. Journal of

476 Geophysical Research: Solid Earth, 93(B7), pp.7729-7740.

477 Dullien, F.A., 2012. Porous media: fluid transport and pore structure. Academic press. 
478 Dutton, S.P. and Willis, B.J., 1998. Comparison of outcrop and subsurface sandstone 479 permeability distribution, lower Cretaceous fall river formation, South Dakota and 480 Wyoming. Journal of Sedimentary Research, 68(5), pp.890-900.

481 Ehrenberg, S.N., 1990. Relationship between diagenesis and reservoir quality in sandstones 482 of the Garn formation, Haltenbanken, mid-Norwegian Continental shelf (1). AAPG bulletin, 483 74(10), pp.1538-1558.

484 Erofeev, A., Orlov, D., Ryzhov, A. and Koroteev, D., 2019. Prediction of porosity and 485 permeability alteration based on machine learning algorithms. Transport in Porous 486 Media, 128(2), pp.677-700.

487 Fisher, A., Rudin, C. and Dominici, F., 2018. Model class reliance: Variable importance 488 measures for any machine learning model class, from the "Rashomon" perspective. arXiv 489 preprint arXiv:1801.01489.

490 Fraley, C., and Raftery, A.E., 2002. Model-based clustering, discriminant analysis and 491 density estimation, Journal of the American Statistical Association, 97(458), pp. 611-631. 492 Frick, T.C., 1962. Petroleum production handbook (Vol. 1). McGraw-Hill.

493 Friedman, J.H., 2001. Greedy function approximation: a gradient boosting machine. Annals 494 of statistics, pp.1189-1232.

495 Ghanbarian, B., Hunt, A.G., Ewing, R.P. and Sahimi, M., 2013. Tortuosity in porous media: a 496 critical review. Soil science society of America journal, 77(5), pp.1461-1477.

497 Gjelberg J., Dreyer, T., Hoie, A., Tjelland, T., and Lilleng, T., 1987. Late Triassic to Mid498 Jurassic sand body development on the Barents and Mid-Norwegian shelf, in J. Brooks and 
499 K. Glennie, eds., Petroleum geology of north west Europe, London, Graham and Trotman, 500 p. $1105-1129$.

501 Huang, S.C., Inman, D.J. and Austin, E.M., 1996. Some design considerations for active and 502 passive constrained layer damping treatments. Smart Materials and Structures, 5(3), p.301.

503 Jensen, J. L., Hinkley, D. V., and Lake, L. W., 1987. A statistical study of reservoir

504 permeability: Distributions, correlations, and averages. SPEFE, 2(6), pp. 461-468.

505 Jensen, J.L., Lake, L.W., Corbett, P.W.M., and Goggin, D.J., 2000. Statistics for petroleum

506 engineers and geoscientists, 2nd ed. Elsevier, Amsterdam, 138 p.

507 Kolodzie Jr, S., 1980. Analysis of pore throat size and use of the Waxman-Smits equation to

508 determine OOIP in Spindle Field, Colorado. Paper SPE 9382 in 55th SPE annual technical

509 conference and exhibition. Society of Petroleum Engineers, 10p.

510 Korvin, G., 1992. Fractal Models in the Earth Sciences. Elsevier, Amsterdam.

511 Kozeny, J., 1927. Soil permeability. Sitzungsber. Oesterr. Akad. Wiss. Wien. Math.

512 Naturwisss. Kl. Abt, 136, p.271.

513 Likas, A., Vlassis, N. and Verbeek, J.J., 2003. The global k-means clustering algorithm.

514 Pattern recognition, 36(2), pp.451-461.

515 Lundberg, S.M. and Lee, S.I., 2017. A unified approach to interpreting model predictions.

516 In Advances in neural information processing systems (pp. 4765-4774).

517 Miller, R. G., 1986, Beyond ANOVA, Basics of Applied Statistics, J. Wiley and Sons, New York, $518317 \mathrm{p}$.

519 Ngo, V. T., Lu, V. D., Nguyen, M. H., Hoang, H. M., Le, V. M., and Son, L., 2015. A comparison of 520 permeability prediction methods using core analysis data. SPE conference paper SPE 
521175650 presented at the SPE Reservoir Characterization and Simulation Conf. and Exhib.,

522 Abu Dhabi, UAE 14-16 Sept. 16p.

523 Panda, M.N. and Lake, L.W., 1994. Estimation of single-phase permeability from parameters

524 of particle-size distribution. AAPG Bulletin, 78(7), pp.1028-1039.

525 Panda, M.N. and Lake, L.W., 1995. A physical model of cementation and its effects on single-

526 phase permeability. AAPG Bulletin, 79(3), pp.431-443.

527 Pettijohn, F. J., 1975, Sedimentary Rocks, Third Ed., Harper and Row, New York, 628p.

528 R Core Team, 2017. R: A language and environment for statistical computing. R Foundation

529 for Statistical Computing, Vienna, Austria. URL http://www.R-project.org/

530 Rezaee, M.R., Jafari, A. and Kazemzadeh, E., 2006. Relationships between permeability,

531 porosity and pore throat size in carbonate rocks using regression analysis and neural

532 networks. Journal of Geophysics and Engineering, 3(4), pp.370-376.

533 Sandrea, R., 2005, Global natural gas reserves - a heuristic viewpoint. Ipc66.com, archived

5344 March 2016. Scheidegger, A.E., 1960, The Physics of Flow Through Porous Media, Revised

535 Ed., University of Toronto Press.

536 Shapley, Lloyd S., 1953. A value for n-person games. Contributions to the Theory of Games

537 2(28), pp. 307-317.

538 Slatt, R. M., 2006. Stratigraphic Reservoir Characterization for Petroleum Geologists,

539 Geophysicists, and Engineers. Elsevier.

540 Scheidegger, A.E., 1960, The Physics of Flow Through Porous Media, Revised Ed., University

541 of Toronto Press.

542 Thomas, S., Corbett, P. and Jensen, J., 1996, January. Permeability and Permeability 
543 Anisotropy Characterisation in the Near Wellbore: A Numerical Model Using the Probe

544 Permeameter and Micro-Resistivity Image Data. In SPWLA 37th Annual Logging Symposium.

545 Society of Petrophysicists and Well-Log Analysts.

546 Walsh, M.P. and Lake, L.W., 2003. A generalized approach to primary hydrocarbon recovery

547 (Vol. 4). Amsterdam: Elsevier. p. 36.

548 Wendt, W. A., Sakurai, S., and Nelson, P. H., 1986. Permeability prediction from well logs

549 using multiple regression. In Reservoir Characterization, L. W. Lake and H. B. Carroll (eds.),

550 Academic Press, pp. 181-221.

551 Wickham, H. (2009) ggplot2: elegant graphics for data analysis. Springer New York.

552 Appendix A. Derivation of a modified Carman-Kozeny equation for

553 uncemented sandstones

554 This section follows the derivation laid out by Panda and Lake (1994).

555 The derivation starts with the Carman-Kozeny equation

556

$$
k=\frac{\phi^{3}}{2 \tau(1-\phi)^{2} a^{2}}
$$

557 where permeability is $k$, porosity is $\phi$, tortuosity is $\tau$, and the specific surface area is $a$. Both

558 the Helium porosity and the interparticle macroporosity have been measured on the Garn

559 data. Klinkenberg-permeability to air is also part of the dataset. To estimate tortuosity and

560 specific surface area, the dataset includes measurements of the median grain size and the

561 Trask sorting coefficient, following the approach proposed by Beard and Weyl (1973). The

562 skewness of the distribution of grain sizes can be extracted from these parameters. 
563 Given this information, a modified Carman Kozeny equation following Panda and Lake

564 (1994) is

565

$$
k=\frac{\bar{D}^{2} \phi^{3}}{72 \tau_{u}(1-\phi)^{2}} \frac{\left(\gamma C_{D}^{3}+3 C_{D}^{2}+1\right)^{2}}{\left(1+C_{D}^{2}\right)^{2}}
$$

566 where $\bar{D}$ is the mean particle size, $C_{D}$ is the coefficient of varation of the particle size

567 distribution $\left(C_{D}=\sigma_{D} / \bar{D}\right), \gamma$ is the skewness of the particle size distribution. and $\tau_{u}$ is the

568 tortuosity of an unconsolidated, uncemented sand.

569 Panda and Lake (1994) do not calculate the original tortuosity. However, there has been a

570 wealth of work on this problem in the physics, soil, and petroleum literature. Several

571 approaches were proposed by Ghanbarian, et al. (2013). Taking their equation 14, coming

572 from Ahmadi et al. (2011) (which assumes monodisperse spheres at hexagonal close

573 packing), original tortuosity follows the equation

574

$$
\tau_{o}=\sqrt{\frac{2 \phi}{3\left[1-B(1-\phi)^{2 / 3}\right]}+\frac{1}{3}}
$$

575 where $B=1.209$.

576 Panda and Lake (1995) use a surface area argument to derive the effective tortuosity for an

577 uncemented sandstone of different size particles, which is

$$
\tau_{u}=\tau_{o}\left(1+C_{D}^{2}\right)
$$

579 The distributions of the grain distribution measures, $\bar{D}, C_{D}, \gamma$, and the tortuosity $\tau_{u}$ are 580 given in Fig. A1. These measures are all highly skewed. 

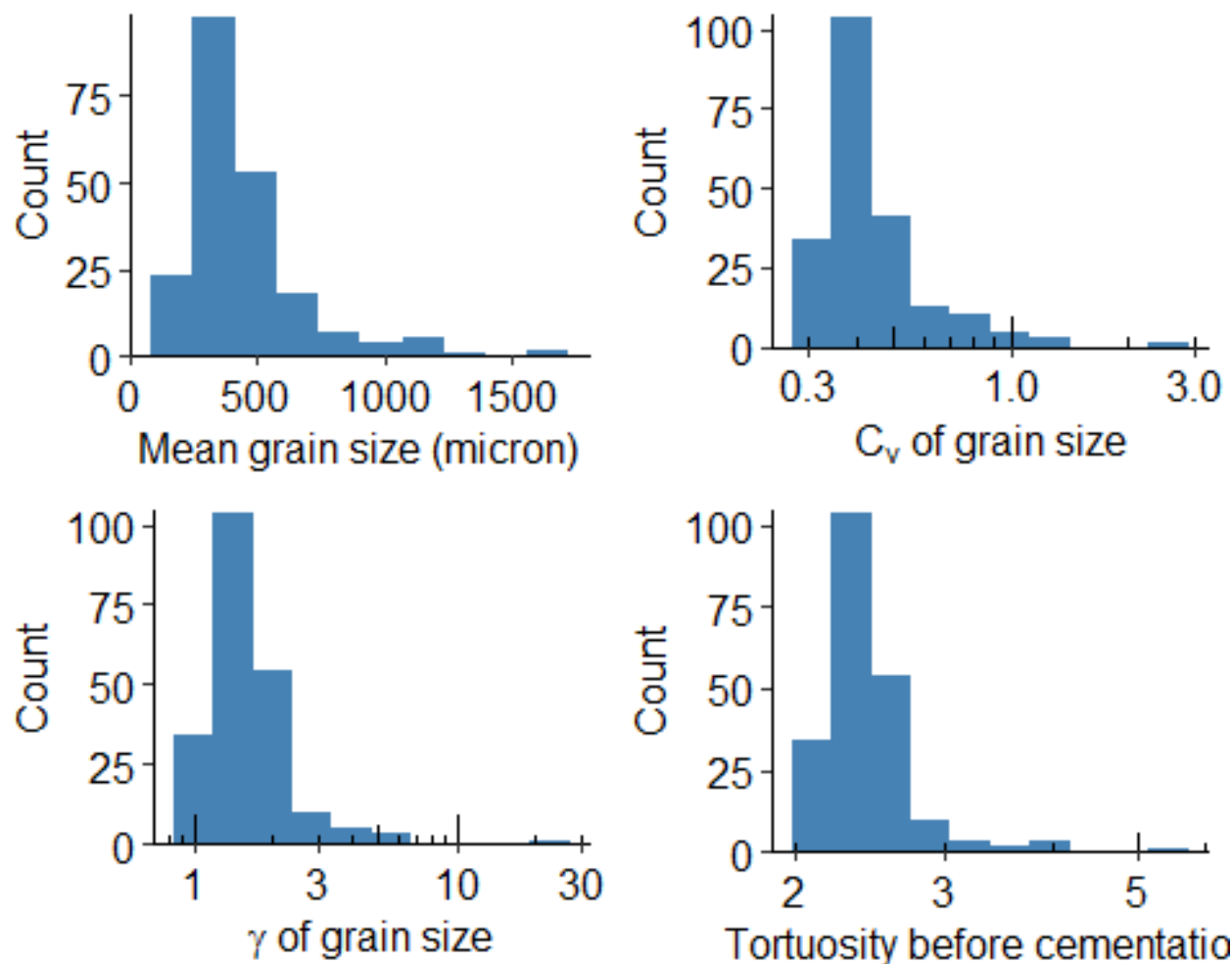

581 Tortuosity before cementatio

582 Figure A1. Histograms of several grain properties.

583 Appendix B: Derivation of Carman-Kozeny corrections for cemented

584 sandstones

585 This section follows the derivation laid out by Panda and Lake (1995).

586 Carman-Kozeny theory does not consider the effect of cementation on permeability, but

587 cement is present in these rocks, and it blocks flow paths, decreasing the rock permeability.

588 In terms of the quantities considered by Carman and Kozeny, this changes the tortuosity

589 and the specific surface area. There are several different cements that are be present, and

590 they are measured through point counting. 
591 Panda and Lake (1995) separate cement types into three categories: pore-filling, pore-

592 lining, and pore-briding, following Neasham (1997). Where cements associate with the

593 pores depends on the thermodynamic properties of the cementing material. Crystal-like

594 kaolinite and dickite cements are pore-filling. Other pore-filling cements include quartz,

595 feldspar, dolomite, and calcite. These cements affect the porosity, but because they do not

596 affect the pore throats or the pore shape, under this model they have a small effect on

597 permeability.

598 Pore-lining cements find it energetically favorable to form long crystals that stretch out

599 from the grains. These cements include the non-kaolinite clay minerals, such as chlorite,

600 illite, and smectite. The long crystals affect permeability more than they affect porosity

601 because of the large surface areas they generate.

602 Pore-bridging cements can partially or completely block the pore throats, decreasing the

603 accessible porosity. This strongly influences the permeability through increasing the

604 tortuosity of the system and decreasing the connectivity. Examples of the minerals that

605 bridge pores include illite, chlorite, and montmorillonite (the non-Kaolin clay minerals).

606 After cementation, the tortuosity and specific surface area has changed. Panda and Lake

607 (1995) suggest an effective tortuosity, $\tau_{e}$, given by

608

$$
\tau_{e}=\tau_{u}\left(1+C_{D}^{2}\right)\left(1+\frac{R m_{b}}{1-m_{b}}\right)^{2}\left(1+\frac{2 m}{(1-m) \phi^{1 / 3}}\right)^{2}
$$

609 where $R$ is a constant equal to 2 indicating the additional distance traveled by the fluid as a

610 function of the thickness of cementation. The volume fraction of pore-bridging cement is 
$611 m_{b}=P_{b}\left(1-\phi_{o}\right) / \phi_{o}$, and the volume fraction of pore-filling cement is $m=P_{f}\left(1-\phi_{o}\right) / \phi_{o}$.

612 ( $\phi_{o}$ is the original porosity of the sandstone grains, before compaction and cementation.)

613 For an unconsolidated sand of variable sizes, the specific surface area is

614

$$
a_{u}=\frac{6\left(\sigma^{2}+\bar{D}^{2}\right)}{\gamma \sigma^{3}+3 \bar{D} \sigma^{2}+\bar{D}^{3}}
$$

615 After cementation, the effective specific surface area follows the equation

$$
a_{e}=a_{u} \frac{1-\phi_{u}}{1-\phi}+a_{b} P_{b}+a_{f} P_{f}
$$

617 where $a_{u}$ is the specific surface area for an unconsolidated, uncemented sand, $\phi_{o}$ is the

618 porosity of an unconsolidated sand, $a_{b}$ is the specific surface area for a pore-bridging

619 cement, $a_{f}$ is the specific surface area for a pore-filling cement, and $P_{b}, P_{f}$ are the relative

620 fractions of pore-bridging and pore-filling cement, respectively.

621 Taking these equations together, the equation for permeability becomes

$$
k=\left[\bar{D}^{2} \phi^{3}\left(\gamma C_{D}^{3}+3 C_{D}^{2}+1\right)^{2}\right]
$$

622

$$
\left\{2 \tau_{e}(1-\phi)^{2}\left[6\left(1+C_{D}^{2}\right) \frac{1-\phi_{u}}{1-\phi}+\left(a_{b} P_{b}+a_{f} P_{f}\right) \bar{D}\left(\gamma C_{D}^{3}+3 C_{D}^{2}+1\right)\right]^{2}\right\}^{-1}
$$

623 Now, with these calculations, the properties of the grain size distribution measured by

624 Ehrenberg (1990) can be used to test the theory derived by Panda and Lake (1995). 


\section{Appendix C: Lognormal distribution statistics}

626 In this appendix we relate median grain size and the Trask Sorting Coefficient $\left(S_{o}\right)$ to the

627 mean, standard deviation, and skewness of the grain size distribution. From the mean and

628 standard deviation, the coefficient of variation, $C_{v}=\bar{D} / \sigma$, can be calculated.

629 Grain size distribution is often described by the median grain size and the Trask Sorting

630 Coefficient $\left(S_{o}\right)$, which is defined by $S_{o}=\sqrt{D_{0.75} / D_{0.25}}$, where $D_{p}$ is the quantile value

631 indicated by $p$, such that $D_{0.25}$ is the 25\%-ile grain size. Panda (1994, Appendix B) derived

632 an equation relating average grain size, Trask Sorting Coefficient, and the standard

633 deviation of the grain size, which is

634 This equation assumes that $D_{p}$ is calculated from the distribution of grain sizes in $\log _{2}$

635 space, but most calculations of $S_{o}$ use the definition provided above, so this should be re-

636 derived.

637 A new derivation, assuming lognormaly distributed grain sizes, can be described with the

638 PDF

639 the mean grain size is $\bar{D}=\exp (\mu+\sigma / 2)$, and in terms of the median and Trask sorting

640 coefficient, the parameters of the distribution are

641

$$
\begin{aligned}
\mu & =\ln D_{0.5} \\
\sigma & =\frac{\ln S_{o}}{\sqrt{2} \operatorname{erf}^{-1}(0.5)}
\end{aligned}
$$

642 Simple R code to test these statistics is given below. It generates numbers from a random 643 lognormal distribution: 
$644 \mathrm{mu}<-3.14159$

645 sigma $<-1$

$646 \mathrm{~d}<-$ rlnorm(10000, mu, sigma) \# distribution of $1 k$ points with mu=pi, sigma=1

647

648 trask <- sqrt(quantile $(d, 0.75) /$ quantile $(d, 0.25))$

649 d_50<- $\operatorname{median}(d)$

650 mu_calc $<-\log \left(d \_50\right)$

651 erfinv <- function( $x)$ qnorm $((x+1) / 2) / \operatorname{sqrt}(2)$

652 sigma_calc <- $\log ($ trask $) /(\operatorname{sqrt}(2) * \operatorname{erfinv}(0.5))$

653 mean_calc $<-\exp \left(\log \left(d \_50\right)+\operatorname{sigma} c a l c / 2\right)$

654 exponent_thingie <- $(2 * \operatorname{sqrt}(2) * \operatorname{erfinv}(0.5))$

655

$656 \operatorname{cat}($

657 "\nThe median is", round(median(d), 1),

658 ". It should be", $\operatorname{round}(\exp (\mathrm{mu}), 1)$,

659 "\nThe mean is", $\operatorname{round}(\operatorname{mean}(d), 1)$,

660 ". It should be", $\operatorname{round}(\exp (m u+\operatorname{sigma} / 2), 1)$,

661 "\nThe standard deviation is", round $(\operatorname{sd}(d), 1)$,

662 ". It should be", round( $\operatorname{sqrt}((\exp (\operatorname{sigma\wedge } 2)-1) * \exp (2 * m u+\operatorname{sigma} 2)))$,

663 "\nThe Trask sorting coefficient is", round(sqrt(quantile(d,0.75) / quan

$664 \operatorname{tile}(d, 0.25)), 2)$,

665 ". InFrom the Trask and median diameters, the mean should be", round(mean_c

666 alc, 1), "or",

667 round(d_50* $\left.\operatorname{trask}^{\wedge}(1 /(2 * \operatorname{sqrt}(2) * \operatorname{erfinv}(0.5))), 1\right)$,

668 ". InThis is a deviation of", round((exp(mu + sigma/2) - mean_calc)/exp (mu 
670

$671)$

$672 \# \#$

673 \#\# The median is 23 . It should be 23.1

674 \#\# The mean is 38.3 . It should be 38.2

675 \#\# The standard deviation is 50.8 . It should be 50

676 \#\# The Trask sorting coefficient is 1.96 .

677 \#\# From the Trask and median diameters, the mean should be 37.9 or 37.9 .

678 \#\# This is a deviation of 0.6 percent

679 The mean grain size can be calculated from the median grain size and standard deviation

680 through the equation (assuming a lognormal distribution of the grain size). In addition, the

681 coefficient of variation and skewness can be calculated. The equations for these terms are

682

$$
\begin{aligned}
\bar{D} & =\exp \left[\ln \left(D_{0.5}\right)+\sigma / 2\right] \\
& =D_{0.5} S_{o}^{1 /\left(2 \sqrt{2} \operatorname{erf}^{-1}(0.5)\right)} \\
& =D_{0.5} S_{o}^{1.349} \\
C_{D} & =\sqrt{e^{\sigma^{2}}-1} \\
& =\sqrt{e^{2.198\left(\ln S_{o}\right)^{2}-1}} \\
\gamma & =\left(e^{\sigma^{2}}+2\right) \sqrt{e^{\sigma^{2}}-1} \\
& =\left(e^{\sigma^{2}}+2\right) C_{D} \\
& =\left(e^{2.198\left(\ln S_{o}\right)^{2}}+2\right) \sqrt{e^{2.198\left(\ln S_{o}\right)^{2}-1}}
\end{aligned}
$$

683 These equations are used in this manuscript to determine the Carman Kozeny coefficients 684 for each sample. 